\title{
THE TYPES OF TUBERCLE BACILLI IN LUPUS AND SCROFULODERMIA
}

\author{
BY THE LATE A. STANLEY GRIFFITH
}

Medical Research Council's Field Laboratories, Milton Road, Cambridge*

I. INTroptction

II. BACTERIOLOGICAL INVESTIGATION OF STRAINS FROM LUPUS

Clinical particulars of the lupus cases

Methods of investigation

Cultural differentiation of strains

Differentiation of strains by pathogenicity

III. Results

Cultural tests

Virulence tests

Guinea-pigs

Bacilli of standard virulence

Bacilli of attenuated virulence

Cultures from guinea-pigs showing no lesions.

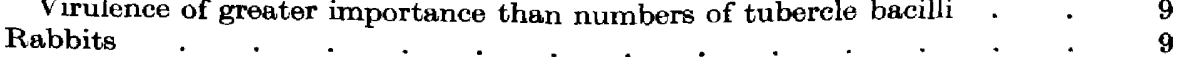

Bovine bacilli of standard virulence $\quad . \quad$. $\quad . \quad . \quad . \quad . \quad . \quad 10$

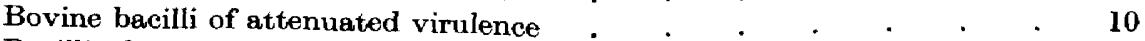

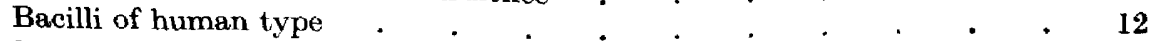

Calves .

Goats .

Monkeys

Summary of virulence tests

Cases of lupus investigated more than once

Passage experiments

Bovine strains

Human strains

Comparison of strains from different lesions excised from the same patient on the same day

Comparison of strains from lupus and from internal tuberculous lesions from

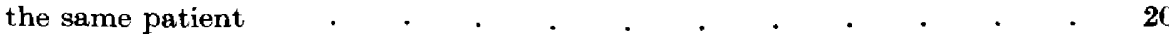

IV. BaCteriological INVESTIGATION OF STRAINS FROM SCROFUlODERMi . . 21

V. Disctession . . . . . . . . . . . . . . . . . . . . 22

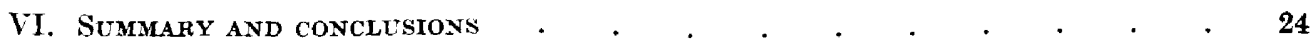

REFERENCES

\section{EDITOR'S NOTE}

Before his death in $1941 \mathrm{Dr}$ A. S. Griffith had prepared a report on strains of tubercle bacilli from cutaneous tuberculosis which he had studied during the past thirty years or so. Strains of this origin are of peculiar interest, because many of

* Requests for reprints should be sent to the Librarian, Central Public Health Laboratory, Colindale Avenue, London, N.W.9. 
them exhibit changes in virulence unlike those met with, except quite occasionally, in other forms of tuberculosis. It was the wish of the late Sir Walter Fletcher that Dr Griffith should prepare a complete account of his investigations for publication in the Special Report Series of the Medical Research Council. Dr Griffith died before his manuscript was quite finished, though all the essential information was included. The report lay untouched till a few months ago when Dr G. S. Wilson offered to prepare an ablureviated version of it suitable for publication in the Journal of Hygiene. In this task he has had to summarize in the text or in short tables the immense amount of tabular matter giving a detailed description of the cases studied and of the reaction of the individual animals into which the strains were injected. It is impossible in such a summary to convey any idea of the enormous labour that Dr Griffith expended in the examination of his original material and in the compilation of his report. Those of our readers wishing for fuller information than is contained in this paper may consult Dr Griffith's original report, which will be kept by the Librarian, Central Public Health Laboratory, Colindale, London, N.W.9.

\section{INTRODUC'TION}

Since 1907 when I* was appointed by the Royal Commission on Tuberculosis (1901-11) to carry out investigations on tubercle bacilli of different origin, I have worked continuously on this subject. Among the earliest strains that I examined was one which had been isolated by Dr Louis Cobbett from a case of lupus vulgaris and which was below the level of standard virulence of bovine strains for calves and rabbits but above that of human strains. Fuller examination showed that its virulence was reduced not only for the calf and rabbit, but also for the pig, goat, monkey and guinea-pig. The anomalous features of this strain were similar to those of certain strains that Dr Fred Griffith and I had obtained from naturally acquired tuberculosis in the horse. Our conclusion on these equine strains was that they were bovine strains which had become modified in virulence during residence in the tissues.

The results with this first lupus strain lod to an extended investigation of the bacteriological characters of tubercle bacilli from lupus and other forms of skin tuberculosis with the twofold object of determining the relative frequency of the different types of tubercle bacilli in these lesions and the degree of variation that these strains exhibited in relation to strains from other forms of tuberculosis. From time to time my results have been published, the last time being in the Zeitschrift für Tuberkulose in 1932, when I reported on 188 strains from lupus. Since then I have examined a further sixteen strains, and the report which I now make refers to the total of 204 strains.

In addition, $I$ include a report on sixty strains isolated from cases of scrofulodermia. To avoid confusion with the lupus strains, these will be described in a separate section towards the end of this paper.

* Throughout this paper the word 'I' refers to Dr Stanley Griffith who prepared the report, and not to Dr Wilson who summarized it. 
A brief summary of the results of examining the total series of strains from lupus and scrofulodermia was included in a paper prepared for the XIth Conference of the International Union against Tuberculosis which was to have been held in Berlin in September 1939 (Griffith, 1941a).

\section{BACTERIOLOGICAL INVESTIGATION OF STRAINS FROM LUPUS}

\section{Clinical particulars of the lupus cases}

Altogether material from 204 patients was studied. The patients were attending light clinics and out-patient departments situated in London or the principal cities of England and Scotland, or were resident patients at the Lord Mayor Treloar Cripples' Hospital at Alton. Numerically, their distribution was as follows:

\section{7 were in London or at Alton \\ 44 were in the north of England \\ 13 were in Scotland \\ $\overline{204}$}

Of the 204 patients, 94 were males and 110 females.

The age distribution was from 2 to 71 years, but $90 \%$ of the patients were children under 15 years.

The duration of the disease, when this was known, was from 3 months to 52 years. Deducting from their ages the previous duration of the disease, one finds that about $80 \%$ of the patients were attacked before the 10th year of life and $94 \%$ before the 20 th year.

Information was available in 152 cases on the part of the body first affected. In 79 cases the disease started on the face or neck, in 34 on the arms, in 31 on the legs, and in 8 on other sites.

A history of tuberculosis other than that of lupus was not usually supplied with the material sent for examination, but in 70 cases the patient was said to have suffered from some other form of tuberculosis-usually cervical adenitis, scrofulodermic abscesses, or tuberculosis of bones and joints-which had appeared before, or less often during, the course of the lupus.

\section{Methods of Investigation}

The original material was either excised skin containing one or more lupus nodules, or scrapings from lupoid patches, or pus obtained by treating lupus nodules with a copper sulphate ointment.

For inoculation the lupoid tissues were triturated in a mortar and suspended in salt solution. With this suspension guinea-pigs-one to three according to the amount of original material - were inoculated, and direct cultures were sometimes sown after treating the suspension with antiformin or potassium hydroxide. In the earlier part of the inquiry most of the guinea-pigs were inoculated intraperitoneally, but later the subcutaneous method was adopted. One of the guineapigs in each set was killed a few weeks after inoculation and cultures were made 
from two or more lymphatic nodes. For this purpose a small portion of the tuberculous tissue was removed aseptically, placed in a small stout test-tube and broken up with a glass rod ground at the end;* a few drops of sterile salt solution were added and the broken-up tissue was brought into suspension. When the node contained pus, a small quantity of this was emulsified directly in a few drops of physiological saline. Three or four plain egg tubes and one $5 \%$ glycerin egg tube were then sown, each with one drop of the suspension, which was spread evenly over the surface. The tubes were incubated at $37 \cdot 5^{\circ} \mathrm{C}$. and the primary colonies, which were generally visible with a lens in from 10 to 12 days and to the naked eye in the third week, were subcultured on solidified bovine serum or plain egg.

\section{Cultural differentiation of strains}

Though it was nearly always possible from the character of the primary colonies on plain egg and $5 \%$ glycerin egg to determine to which type, human or bovine, a given strain belonged, each strain was in fact tested on a series of media, namely, plain egg and plain borine serum, and egg, serum, agar, potato and broth made up with $5 \%$ glycerin. These media were inoculated from plain egg or serum cultures-never from cultures containing glycerin.

The cultural differentiation of human and bovine types of tubercle bacilli in their normal and variant forms has been described in the reports of the Royal Commission (Report, 1907, 1911), and in some of my own papers, to which reference may be made (Griffith, 1914 $a, 1915,1920$, I922, 1924, 1941 $b$ ). Suffice it to say here that all strains of human type grow well (eugonically) on egg and bovine serum, the growth, however, being improved by the addition of $5 \%$ glycerin. On plain egg growth is normally visible in 10-12 days. Most strains likewise give a eugonic growth on $5 \%$ glycerinated agar, potato and broth, but a very small minority, though growing well on plain and glycerinated egg and serum, give only a poor (dysgonic) growth on glycerinated agar, potato and broth. These are known as dysgonic human strains.

Bovine strains, on the other hand, though growing at the same rate as human strains, always grow less luxuriantly on plain egg and plain bovine serum. Hence they are referred to as dysgonic. The addition of $5 \%$ glycerin may inhibit their growth, may have no effect on it, or may even improve it. When colonies of the second or third type are subcultured, they are found to grow readily on glycerincontaining media, whereas the plain egg strain retains its distinctive dysgonic characters. It is for this reason that, when testing strains on a series of media for their cultural behaviour, one should always inoculate the tubes from a plain nonglycerinated egg or serum culture.

The cultural distinction between a dysgonic human strain and a bovine strain that grows well on glycerin egg is aided by the canary yellow pigment formed by the human strain on suitably coloured batches of bovine serum.

\footnotetext{
* Esually known as a Ciriffith tube.
} 


\section{Differentiation of strains by pathogenicity}

The primary cultures obtained on plain egg medium were tested for their virulence by injection into guinea-pigs and rabbits. (A few exceptions were made for characteristically human eugonic strains that appeared, from lesions produced in guinea-pigs inoculated with the original material, to be fully virulent.) Many strains, mainly of the dysgonic type, were tested in addition in the calf, goat and monkey, and a few also in the pig, baboon and fowl. Some of the animals were allowed to die; some were killed after a few weeks or months; and some were left for over a year. The number of animals injected with any given strain varied considerably. With some of the attenuated strains repeated tests were made on different animals, particularly when the results in animals of the same species lacked uniformity, or when apparently discrepant results were obtained between animals of different species.

\section{RESULTS}

\section{Cultural tests}

With 5 exceptions the strains from the 204 lupus patients could be classified readily into two groups according to their cultural characters (Table 1, p. 15). In one group the cultures were eugonic, growing luxuriantly on all the differential media and being identical in their general characters with the common human tubercle bacillus; these accounted for 98 of the 204 strains. In the other group the cultures grew less luxuriantly than the human type of bacillus and were identical culturally with tubercle bacilli of bovine tuberculosis; these were 101 in number and are referred to as dysgonic bovine strains. Since many of this latter group were less virulent for rabbits, goats and calves than typical bovine strains-some indeed producing after subcutaneous inoculation no more disease than human bacilligreat pains were taken to establish definitely their cultural characters. They were tested repeatedly, both in early generation and after they had been a long time in cultivation, until it was certain that none of them was a human strain that was slow in adapting itself to typical growth on artificial media.

There remain five cases from which the cultures did not conform exactly to either type. Four of the strains grew luxuriantly on egg, bovine serum, glycerin egg and glycerin serum, the growths on serum exhibiting a yellow pigment; but on glycerin agar, glycerin potato and glycerin broth the growths were poor. These strains are designated dysgonic human.

The fifth strain grew moderately well on all media, particularly glycerin agar and glycerin potato; growth was distinctly better than that of any bovine strain but less luxuriant than that of the eugonic human type of bacillus. Cultures on bovine serum were not pigmented. This strain is referred to as a eugonic bovine.

Though, as will be mentioned later, strains differing in virulence were sometimes isolated from the same patient, in no instance were different cultural types isolated. That is to say, there were no cases in which human and bovine strains were found together. 


\section{VIRULENCE TESTS}

Space does not permit a full record of the numerous experiments carried out on different animals to determine the virulence of the strains. All that can be done here is to summarize and comment on the results obtained.

\section{Guinea-pigs}

The guinea-pig is highly susceptible to infection with mammalian tubercle bacilli and invariably develops progressive general tuberculosis after inoculation with even very small doses of virulent bacilli, whether of bovine or human type. On the average the bovine type is more virulent for the guinea-pig than the human type, giving rise to a more acute disease that causes death sooner than the human bacillus. In guinea-pigs infected with human bacilli the lymphatic nodes do not generally become so large or so caseous as those infected with bovine bacilli, and there is often less necrosis in the visceral lesions. These differences, however, are not always present and cannot therefore be relied upon to distinguish bovine from human bacilli.

In this work guinea-pigs were injected as a rule with $0.1 \mathrm{mg}$. of moist culture intraperitoneally or, more often, subcutaneously, the needle in this latter method being introduced into the lumbar region and directed downwards and outwards towards the flank.

\section{Bacilli of standard virulence}

The reactions of these animals to injection with $0.1 \mathrm{mg}$, or less often $1 \mathrm{mg}$., of moist virulent bacilli fell into three groups: (a) Acute general tuberculosis: this form occurred after intraperitoneal inoculation; the animals died within about 3 weeks, and showed a severe peritoneal reaction with effusion of fluid into the pleural cavities, just visible lesions in the spleen and liver, and small areas of caseation in the cortex of some abdominal and thoracic lymph nodes. (b) Caseonecrotic general tuberculosis: this was the characteristic form of tuberculosis seen after subcutaneous inoculation with $0.1 \mathrm{mg}$.; the animals died in 6-12 weeks and at post-mortem showed enlargement and extensive caseation of most of the lymphatic nodes, extensive necrosis of the liver and spleen giving these organs a marbled or mottled appearance, and moderate numbers of miliary caseating tubercles in the lungs. As a rule guinea-pigs infected with virulent bovine bacilli tended to die earlier than animals injected with virulent human bacilli, and showed after equal intervals of time rather more extensive caseation, particularly of the lymphatic nodes. (c) Chronic general tuberculosis: this was the form seen in animals surviving for more than 3 months. The lesions were very variable; they were usually characterized by fibrosis, and by less extensive caseation and necrosis than in guinea-pigs dying earlier. The spleen was sometimes greatly enlarged, dark red in colour, with a greyish mottling and discrete tubercles, or composed almost entirely of dense creamy-white tissue. The liver was enlarged, pale, fatty, irregular on the surface, and mottled with irregular bile-stained areas of necrosis, or dense 
and fibroid containing cysts filled with thin greenish pus. In some instances the immediate cause of death was haemorrhage from the swollen spleen or liver into the peritoneal cavity. The lungs contained either discrete tubercles and nodules or else showed the conversion of a lobe, in whole or in part, into fibroid tissue containing small cavities. The lymphatic nodes were dense and fibroid, sometimes dotted with calcareous grains, sometimes containing cavities filled with thin pus, and sometimes converted into caseous abscesses. Tuberculous lesions were rare in the kidneys; they were seen only once in a joint and never in the eyes.

\section{Bacilli of attenuated virulence}

The pathogenic effects of these bacilli varied widely from chronic fibrotic general tuberculosis to retrogressive tuberculosis limited to the seat of inoculation and the nearest lymphatic nodes. Most of the strains gave rise to disease of one of the following five types: $(d)$ Chronic general tuberculosis: this form resembled in anatomical distribution and macroscopic features the chronic form $(c)$ already described, but the duration of life was longer and the lesions showed more advanced fibrosis. Whereas guinea-pigs that developed chronic general tuberculosis after injection with virulent tubercle bacilli seldom lived longer than 100 days, those injected with attenuated bacilli did not die for a year or more. (e) Atypical subacute general tuberculosis : this form was observed in guinea-pigs dying in from 5 to 10 weeks after intraperitoneal inoculation. The omentum was either slightly retracted and thickened along its free margin or contained discrete tubercles; the mesentery was normal except for a few grey points; the peritoneum showed a few caseous tubercles in the lumbar region. The spleen was only moderately enlarged and was red speckled with grey points. The liver was of normal size and colour and was speckled with grey or sometimes yellowish foci. The lungs were crepitant, and showed thickening of the interlobular septa but no definite tubercles. The kidneys frequently contained tubercles, situated usually in the outer margin of the medullary zone. There was slight excess of fluid in the pleural and peritoneal cavities. The lymphatic nodes, except perhaps the sternal, omental and portal which might contain caseous foci, were normal in size or only slightly enlarged and free from caseation. Caseous tubercles were always present in the areolar tissues of the groins, axillae, neck and lumbar regions. The chief points of distinction between this form of tuberculosis and that produced by standard bacilli were the slower course of the disease, the less intense peritoneal reaction, the absence or slight amount of necrosis in the organs and glands, the frequent presence of tubercles in the kidneys, and the occurrence of tubercles in the areolar tissues. ( $f$ ) Atypical chronic general tuberculosis: in this form, which followed subcutaneous or sometimes intraperitoneal inoculation, the distribution of the disease was the same as in $(c)$, but the lesions in the organs were less extensive and more discrete, showed little or no evidence of previous caseo-necrosis, and appeared everywhere to be in a state of retrogression. The lymphatic nodes, except the nearest, were dense, fibrous and sometimes of cartilaginous hardness; they were homogeneous throughout or contained calcareous grains or little pockets of thin pus. 
The spleen was largely composed of dense white fibrous tissue, or contained fibrocalcareous tubercles, or was atrophic and cicatricial. In the liver there were grey fibrous tubercles, or else nodules or areas containing cysts filled with thin greenish yellow pus. The lungs contained fibrous areas and tubercles. Many of the animals when killed 212-385 days after inoculation were fat and in good general condition. (g) Slight retrogressive genersl tuberculosis: in this form the lesions were few and retrogressive. In animals inoculated intraperitoneally there were scanty discrete tubercles in the omentum; in those inoculated subcutaneously the local lesion was represented by a scar. Disseminated lesions in the form of grey fibrous tubercles, occasionally gritty, were sometimes limited to the spleen, but were occasionally present also in the liver and lungs. The lymphatic nodes, usually the portal, bronchial and iliac, were a little enlarged and sometimes showed calcareous grains, but occasionally all the nodes except the regional were practically normal. A few of the guinea-pigs died of causes other than tuberculosis: the rest, when killed after periods up to 917 days, were fat and had gained greatly in weight. $(h)$ Local tuberculosis : in this form the lesions were limited to the seat of inoculation and the nearest lymphatic nodes.

Of the total of 102 culturally human strains of tubercle bacilli, 41 proved to be of standard virulence for the guinea-pig: the remaining 61 were attenuated to a greater or less degree. Three of this latter group yielded rather equivocal results in the guinea-pig, but were found by injection into monkeys to be in an early stage of attenuation.

Of the total of 102 culturally bovine strains, 34 proved to be of standard virulence for the guinea-pig and 68 of attenuated virulence. Six strains of this latter group proved fully virulent for the guinea-pig, but were found on injection into other species of animals to be slightly below the standard level of virulence.

Just as virulent bovine bacilli gave rise to a rather more acute and severe disease in guinea-pigs than virulent human bacilli, so bovine bacilli of attenuated virulence proved more pathogenic on the whole than attenuated human bacilli.

With the attenuated group many strains produced different forms of tuberculosis in different guinea-pigs inoculated by the satme route: or a failure was seen in the usual correspondence between the forms of tuberculosis resulting from inoculation by different routes. In these instances the strains were assigned their places in accordance with the results produced in the majority of the guinea-pigs inoculated.

In general conclusion it may be noted that the guinea-pig is of special value for detecting attenuation in the human type of bacillus, since there is no other animal except the monkey which can be relied upon for this purpose.

\section{Cultures from guinea-pigs shouing no lesions}

With material from 20 patients infected with attenuated bacilli, 11 of human and 9 of bovine type, cultures of tubercle bacilli were obtained from guinea-pigs showing no evidence of disease. Most of these guinea-pigs were examined from 21 to 58 days after inoculation, but two of them were not killed for 204 and 664 days. From both of these animals a suspension of the inguinal lymph nodes 
yielded colonies-in the second instance only four-of tubercle bacilli. It is abundantly clear from these results that the absence of macroscopic tuberculosis in a guinea-pig injected with lupus tissue does not necessarily exclude the presence of tubercle bacilli in the inoculum. To be certain that the material is non-infective, one should always make cultures from the lymph nodes adjacent to the seat of injection, even though these show no obvious sign of tuberculosis.

\section{Virulence of greater importance than numbers of tubercle bacilli}

The truth of this statement may be illustrated by the following two examples. A suspension of lupus material was injected intraperitoneally into three guineapigs; one of them developed general tuberculosis, but the other two showed no lesions and cultures from them remained sterile. A culture from the first guineapig proved to be a fully virulent bovine strain. As each guinea-pig was given approximately equal amounts of the suspension, the animal that became infected must have received an exceedingly small number of tubercle bacilli. On the other hand, a suspension from another case of lupus that contained comparatively numerous tubercle bacilli gave rise to no more than slight retrogressive disseminated tuberculosis; the animal was killed after 408 days, and the tubercle bacilli that were recovered proved on further examination to be definitely attenuated.

\section{Rabbits}

The Royal Commission on Tuberculosis found that for distinguishing human from bovine bacilli the rabbit could be substituted for the calf, provided the correct dosage was used- $-0.01 \mathrm{mg}$. intravenously or $10 \mathrm{mg}$. subcutaneously. Unlike the calf, the rabbit does not always exhibit high resistance to infection with human bacilli: it may develop progressive general tuberculosis after intravenous, and even after subcutaneous, inoculation. A severe result is, however, unusual after subcutaneous inoculation, and with experience is readily distinguished from the more chronic forms of general tuberculosis occasionally produced by bovine bacilli. On the other hand, after intravenous or intraperitoneal injection of relatively large doses of human bacilli- 0.1 or $1.0 \mathrm{mg}$ - - an acute tuberculosis may follow that is difficult to distinguish from that caused by bacilli of bovine type. Sometimes a rabbit having survived the acute stage may succumb later to chronic progressive generalized tuberculosis. The intravenous and intraperitoneal routes should therefore be used with caution and, when an equivocal result is obtained, the test should be repeated and controlled by subcutaneous inoculation. It is advisable also to isolate the organism from the severely affected rabbit and ascertain its characters and virulence in order to exclude the possibility of infection with a mixture of human and bovine bacilli.

Lupus strains were tested by the injection of $0.01,0.1$ or $1 \mathrm{mg}$. intravenously, or of $10 \mathrm{mg}$. or more subcutaneously in the back midway between the scapulae. 


\section{Bovine bacilli of standard virulence}

Intravenous injection of $0.01 \mathrm{mg}$. of moist virulent bacilli classified culturally as of the bovine type led to death from general miliary tuberculosis in from 20 to 35 days. On post-mortem examination the lungs filled the chest, did not collapse, and were closely beset with miliary caseating tubercles coalescent in places. The tracheal lymph nodes were enlarged, oedematous and partly caseous. The liver and the greatly enlarged spleen were peppered with minute grey tubercles. The kidneys showed a moderate number of tubercles in the cortices. All the lymphatic nodes and the rib marrow contained tubercles.

Subcutaneous injection of $10 \mathrm{mg}$. of moist virulent bovine bacilli was followed by progressive generalized tuberculosis causing death in 23-100 days. The postmortem appearances varied with the duration of life. Rabbits dying within 5 weeks showed a generalized miliary tuberculosis similar to that produced by intravenous inoculation. In rabbits dying in 6-8 weeks the disease still had a miliary character, but the tubercles in the lungs and kidneys were larger and more advanced in caseation, showing a tendency to coalesce in the lungs and to project from the kidneys. Tubercles in the liver and spleen were less numerous than in the more acutely affected animals. In rabbits dying in from 8 to 14 weeks the tuberculous lesions had a characteristic aspect and distribution. The lungs were large, filled the thoracic cavity, and were almost entirely composed of coalescent caseating nodules and masses. The kidney cortices were beset with caseating nodules of varying size up to that of a large pea, the majority of them projecting prominently from the surface and penetrating deeply into the kidney substance in a wedgeshaped manner. Caseous tubercles were generally numerous in the submucous tissue of the appendix and sacculus; they were present in varying number in the omentum, and were found occasionally in the bone marrow and the popliteal lymph nodes. The liver and spleen, on the other hand, were generally free from lesions.

\section{Bovine bacilli of attenuated virulence}

These strains were less virulent for the rabbit than typical bovine strains, and they varied widely among themselves in the degree to which they fell below standard. Some strains inoculated intravenously caused acute and rapidly fatal general miliary tuberculosis equal in severity to that produced by bovine bacilli of standard virulence, but after subcutaneous inoculation proved distinctly less virulent than typical bovine bacilli. At the other end of the scale of virulence there were strains which when inoculated subcutaneously proved no more virulent than the human type of tubercle bacillus but which after intravenous inoculation were definitely more pathogenic than human bacilli. Owing to the frequent lack of correspondence between the results of intravenous and subcutaneous inoculation, it has not been possible to arrange the attenuated bovine strains in any exact order of virulence for rabbits; they have, however, been roughly divided into three groups according as their average virulence fell slightly, moderately, or greatly below that of standard bovine bacilli. 
The group of slightly attenuated bacilli gave rise on intravenous inoculation to acute or subacute general miliary tuberculosis, though not always in every rabbit. The group of moderately attenuated bacilli gave rise to severe tuberculosis which, however, did not prove fatal within the usual time. Most of the strains belonging to the greatly attenuated group caused a chronic form of tuberculosis which did not prove fatal for several months. At post-mortem examination of these animals the lungs were sometimes massively tuberculous, but more often they contained discrete or semi-confluent tubercles showing retrogression, together with tracts of tuberculous tissue around the margins of all the lobes and along the dorsal borders of the caudal lobes. The spleen was much enlarged and beset with caseous or easeo-calcareous tubercles. The liver contained small grey tubercles. The kidneys were usually pitted and scarred on the surface; on section caseo-purulent streaks were seen to extend from the cortex to the apices of the papillae; signs of repair were generally evident. In many of the chronically affected animals lesions were present in nearly every part of the body-eyes, lachrymal glands, bones, joints, testes, skin, subcutaneous tissues, omentum and appendix. The long survival of the animals is attributable to an arrest in development of the lesions in the great organs, particularly the lungs, an arrest that is not observed after inoculation of fully virulent bacilli.

Subcutaneous inoculation gave rise to very diverse pathological effects ranging from progressive general tuberculosis to tuberculosis limited to the seat of injection and the regional lymphatic nodes. The local lesion produced was usually a thinwalled cyst with caseous or caseo-purulent contents; sometimes it was firm, sometimes ulcerated. The nearest lymphatic nodes were much enlarged, caseous and softened; sometimes they were partly caseous or contained gritty caseous nodules or tubercles. In the more severely affected rabbits the lungs and kidneys bore the brunt of the disease. The lungs were either massively tuberculous, or contained moderate numbers of firm caseating tubercles, or showed tracts of caseating tissue along the thin margins of the lobes and the dorsal borders of the caudal lobes with occasional discrete tubercles in the substance, or in very chronic cases they contained cavities or calcareous lesions. The kidneys sometimes showed extensive replacement of the cortex with the nail- or wedge-shaped nodules that are characteristic of infection with bovine bacilli; the nodules were sometimes as much as $1 \mathrm{~cm}$. across and often had a mulberry-like configuration. In other animals the kidneys were pitted and scarred on the surface, and contained caseous tubercles in the cortex, caseous striae in the medulla, and caseous pus in the pelvis. Other structures frequently found to be tuberculous were the spleen, serous surfaces, appendix, bone marrow, lymphatic nodes and, in animals surviving longer than 3 months, the joints and eyes. In most of the rabbits injected with attenuated bovine strains the lesions in the lungs and kidneys were slight, resembling those occasionally produced by strains of human type; but, whereas human tubercle bacilli inoculated subcutaneously seldom give rise to disease in other organs, the attenuated bovine strains almost invariably gave rise in addition to lesions in one or more of the other organs and tissues mentioned above. 


\section{Bacilli of human type}

Of the 102 culturally human strains, 70 were tested intravenously on 129 rabbits. The dose varied from 0.01 to $9 \mathrm{mg}$. A few of the rabbits died of tuberculosis after the larger doses, but most of the animals were killed in good condition after 90-429 days. Three animals dying early after $1.0 \mathrm{mg}$. showed generalized tuberculosis. Fifteen animals that died or were killed between 2 and 6 months showed severe to moderately severe chronic tuberculosis, either generalized or limited to the lungs, which showed caseating pneumonia. In 59 animals slight tuberculosis of the lungs and kidneys was present, with, in some instances. a few tubercles in other organs, such as the spleen, lymphatic nodes, joints, mammae or testes. In 35 rabbits only one organ--lungs, kidneys or spleen-was the seat of minimal lesions, sometimes no more than a single tubercle being seen. In the remaining 17 rabbits careful search failed to reveal the existence of any macroscopic lesions.

Of the 102 culturally human strains 71 were tested subcutaneously on 143 rabbits, the dose ranging from 10 to $57 \mathrm{mg}$. One animal, which had been injected with $50 \mathrm{mg}$., died of tuberculosis in 89 days. A few died from other causes. The rest were killed in good condition in 90-430 days. Of the 143 rabbits, 81 showed disease confined to the site of inoculation or with slight involvement of the neighbouring scapular nodes. In 6 rabbits there was moderate tuberculosis of the lungs; in the remainder the lung disease was trivial, the lesions being confined mainly to discrete tubercles. One or two tubercles were found in the kidneys of 19 of the animals. No organs other than the lungs and kidneys were affected.

\section{Calves}

The Royal Commission on Tuberculosis found that tubercle bacilli of human type inoculated into the subcutaneous tissues were incapable of setting up progressive tuberculosis in the calf, however large the dose of bacilli introduced. The bovine tubercle bacillus, on the other hand, almost invariably gave rise to rapidly fatal progressive generalized tuberculosis when a large dose was inoculated subcutaneously. To small doses of bovine bacilli the calf frequently offered considerable resistance and developed only a limited or slightly generalized tuberculosis with retrogressive features. Experience showed that a dose of $50 \mathrm{mg}$. of young serum cultures of bovine bacilli was almost always sufficient to produce generalized progressive tuberculosis proving fatal within 12 weeks. Even $200 \mathrm{mg}$. of human bacilli, however, never produced progressive tuberculosis : and, when killed after 3 months, the animals showed no more than lesions at the site of inoculation and in the nearest lymph nodes, or minute retrogressive lesions scattered in some organs and glands.

In this study the usual dose given was $50 \mathrm{mg}$. subcutaneously, but with some strains this was increased to $100 \mathrm{mg}$.

Culturally bovine strains from 19 cases of lupus were injected subcutaneously into 41 calves. The pathogenic effects ranged from severe and rapidly fatal general tuberculosis to tuberculosis limited to the seat of inoculation and nearest glands. According to the severity of the disease produced, the strains could be divided into five groups: 
Group 1 comprised 3 strains that produced rapidly fatal general tuberculosis in all the calves inoculated, the animals dying in 17-40 days.

Group 2 comprised 4 strains that produced rapidly fatal general tuberculosis in one or more calves and disease of less severity in another not proving fatal within the period of observation, 94-107 days. Experiments on other animals showed that 2 of the strains were fully virulent and 2 less virulent than standard bovine tubercle bacilli.

Group 3 comprised 6 strains that produced general tuberculosis of no great severity, with lesions exhibiting retrogressive features.

Groups 4 and 5 comprised 6 strains that produced respectively either slight disseminated retrogressive tuberculosis or tuberculosis limited to the seat of inoculation and the adjacent lymph node. The effects produced by these strains were hardly distinguishable from those produced by tubercle bacilli of human type.

Culturally human strains from 12 cases were injected subcutaneously into 13 calves. Apart from one calf that died of non-tuberculous renal disease 26 days later, the animals were killed in good condition after 95-123 days. Five of the strains produced slight disseminated tuberculosis, 5 produced a local lesion with slight retrogressive disease of the prescapular gland, and 2 gave rise to a local lesion only.

\section{Goats}

The goat, though rarely the subject of natural tuberculosis, can readily be infected experimentally with bovine bacilli. To this type of bacillus the young goat is more susceptible than the calf, or even the rabbit. To tubercle bacilli of human type, on the other hand, the goat is highly resistant and never develops, as the rabbit occasionally does, slowly progressive tuberculosis after subcutaneous inoculation of large doses. For these reasons the goat is an excellent substitute for the calf, and, moreover, is much less expensive to buy and maintain.

The disease in goats acutely infected with bovine bacilli is characteristic. At the seat of inoculation there is an ulcerated caseo-necrotic lesion. The adjacent lymph nodes-prescapular and lower cervical-are much enlarged and extensively caseated. The lungs are large, consolidated and closely beset with miliary tubercles. There is cavitation in the caudal lobes, most advanced postero-dorsally; and in animals dying of tuberculosis 6 weeks after inoculation the lung tissue may be riddled with small intercommunicating cavities, the bronchi and trachea containing caseo-pus rich in tubercle bacilli. The spleen, liver and kidneys contain miliary tubercles, but these organs are much less severely affected than the lungs. All the lymphatic nodes may contain tubercles; in the thoracic nodes the tubercles are numerous and confluent. In more chronic cases the lungs are always the most severely affected of the organs and often contain cavities.

Culturally bovine strains from 28 cases of lupus were injected subcutaneously into 55 goats; the usual dose was $10 \mathrm{mg}$., but some of the less virulent strains were tested in doses up to $50 \mathrm{mg}$. The effects produced were very diverse and varied from rapidly fatal progressive general tuberculosis to slight generalized retrogressive 
tuberculosis. The period of observation ranged from 26 to 402 days. Five groups were recognized:

Group 1 comprised 3 strains that gave rise to general miliary tuberculosis proving fatal in 26-43 days.

Group 2 comprised 8 strains that produced severe and fatal generalized tuberculosis of a duration longer, however, than in group 1.

Group 3 comprised 6 strains each of which produced fatal tuberculosis in one goat, the animals dying in 3l-75 days, and disease of less severity, not proving fatal within a period of $94-253$ days, in another.

Group 4 comprised 4 strains that gave rise to a chronic form of general tuberculosis which was either not fatal in 95-402 days, or caused death in 107-184 days. In most of the goats that were killed the disease was slowly progressive, and the animals would eventually have died of the disease.

Group 5 comprised 7 strains that caused only moderate or slight general retrogressive tuberculosis not proving fatal in 107-221 days. Except in one goat, the disease produced by this group of strains was more widespread than that in goats inoculated with human tubercle bacilli.

\section{Monkeys}

Of all the species of animals used in the laboratory those belonging to the order Primates have proved the most susceptible to experimental infection with tuberculosis. In these species an extremely small dose of virulent tubercle bacilli, whether of bovine or human type, inoculated subcutaneously always causes progressive general tuberculosis.

In this work Macacus rhesus was used, and injections of lupus strains were made subcutaneously into the tissues of the back, as a rule with $1 \mathrm{mg}$., but occasionally with doses as small as $0.00001 \mathrm{mg}$., or as large as $10 \mathrm{mg}$.

Strains from 30 cases were injected subcutaneously into a total of 88 monkeys (Macacus rhesus). Four human and 3 bovine strains gave rise to acute generalized tuberculosis characteristic of fully virulent bacilli proving fatal in 4-8 weeks. Twelve human and 11 bovine strains proved to be below standard virulence, causing disease that ranged from generalized tuberculosis with delayed death to chronic progressive tuberculosis, the animals dying in between 50 and 413 days after inoculation. Even the least virulent strain proved capable of setting up fatal general tuberculosis.

Post-mortem examination on animals that died of the acute form of the disease revealed a local abscess which had sometimes ruptured; enlargement and caseation of the axillary, vertebral and deep cervical lymph nodes : an enlarged spleen closely beset with caseous softened nodules up to $3 \mathrm{~mm}$. in diameter; an enlarged liver studded with miliary caseous tubercles; a variable number of shotty miliary tubercles in the lungs; only a small number of tubercles in the kidneys; caseous tubercles or small caseous areas in all the lymphatic nodes; tubercles in the omentum and sometimes in other organs, such as the bone marrow and muscles. In monkeys that lived up to 8 weeks the distribution of the disease was the same, 
but the lesions in the organs and glands were generally larger and in the lungs usually more numerous.

Animals dying of chronic tuberculosis showed a wider distribution of lesions than those seen in the acute form. In addition to the organs already mentioned, the pleura, brain, subcutaneous tissues, flat bones of the skull, suprarenals, tongue, heart and voluntary muscles were often affected, and less often the thyroid, pancreas, pericardium, skin, eye, vocal cords, testes and ribs. The joints and meninges were spared. Cerebral lesions were quite frequent; the monkeys sometimes became blind several weeks before death, the result of caseous abscesses in the occipital lobes. In 2 monkeys the eyes were affected directly, one having a miliary tubercle in the iris, the other showing destruction apparently from extension of a tuberculous abscess at the back of the orbit. Skin lesions were in the form of pustules rich in tubercle bacilli. Lesions of the tongue originated in the submucous tissues and were generally ulcerated. Caseous lesions were also occasionally found in the walls of the stomach and intestines. Of the chief organs the spleen bore the brunt of the disease most heavily; sometimes it was almost completely replaced by softened caseous material. The liver, lungs and kidneys in chronic cases showed a varying, but never very large, number of caseous tubercles and nodules.

Attenuated human strains proved to be, on the average, less virulent than attenuated bovine strains.

\section{Summary of virulence tests}

The results of the virulence tests are summarized in Table 1. In allocating a strain to its particular group attention was paid to the sum total of results on all the animals into which it was injected. With attenuated strains discrepancies occurred sometimes in the different animals of the same species, depending presumably upon the varying susceptibility of the individual animals, and sometimes in animals of different species, depending to some extent on differences in reaction to human and bovine bacilli.

Table 1. Distribution of human and bovine types of standard and of attenuated virulence

\begin{tabular}{|c|c|c|c|c|}
\hline \multirow[b]{2}{*}{ Cultural type } & \multicolumn{4}{|c|}{ Virulence } \\
\hline & Standard & At tenuated & Total & $\begin{array}{l}\text { Percentage } \\
\text { attenuated }\end{array}$ \\
\hline Human eugonic & 39 & $\mathbf{5 9}$ & 98) & $59 \cdot 8$ \\
\hline Human dysgonic & 2 & 2 & 4 ) & 08.8 \\
\hline Bovine dysgonic & 34 & 67 & $101\}$ & $66 \cdot 7$ \\
\hline Bovine eugonic & 0 & 1 & 15 & 00.7 \\
\hline Total & 75 & 129 & 204 & $63 \cdot 2$ \\
\hline
\end{tabular}

Neither the guinea-pig nor the rhesus monkey distinguishes clearly between the two types, but they do show whether a strain of either type is fully virulent or not.

The calf, the goat and the rabbit all distinguish bovine strains of full or moderately attenuated virulence from human strains; but with greatly attenuated bovine 
strains these animals are of unequal value for differential purposes. On account of its relatively high natural resistance to tuberculosis the calf is the least suitable for distinguishing greatly attenuated bovine from human strains. The rabbit has the disadvantage of being not always resistant to bacilli of human type; it may develop an acute or slowly progressive disease after intravenous inoculation, and moderate pulmonary or slight disseminated tuberculosis after subcutaneous inoculation. Attenuated bovine strains inoculated subcutaneously often give rise to no more disease than that produced by human strains. If the subcutaneous test was the only one applied to these strains one would necessarily have to regard them as of human type, but when they are injected intravenously they generally produce effects that are more severe than those caused by human strains. The subcutaneous test on the rabbit can stand alone only when the result is the severe progressive generalized tuberculosis characteristic of standard bovine strains. Intravenous inoculation, on the other hand, can stand alone only when the result is minimal.

Rapidly fatal general miliary tuberculosis, though implying high virulence, does not necessarily imply full virulence, since in the present series several bovine strains of modified virulence nevertheless gave rise to this form of tuberculosis after intravenous injection. Both subcutaneous and intravenous injection should therefore be used in the rabbit, since the intravenous method alone may fail to detect minor degrees of attenuation, and the subcutaneous method alone may fail to distinguish between the human and the bovine types of bacillus.

The goat, which possesses higher and apparently more uniform susceptibility to standard bovine strains than the calf and is more resistant to the human bacillus than the rabbit, has proved the most reliable species for distinguishing attenuated bovine from human strains. In only one instance did a goat inoculated with an attenuated bovine strain give an equivocal result.

It may perhaps be noted that in the few experiments undertaken the dysgonic human strains behaved similarly in virulence to the eugonic human strains, two being of standard and two of attenuated virulence. Subsequent observations, which require confirmation. suggested, however, that in the field vole (Microtus agrestis) dysgonic human strains were rather more virulent than eugonic human strains (Griffith, 194la).

\section{Cases of lupus investigated more than ance}

Many of the cases of lupus were repeatedly investigated with the object of ascertaining whether or not tubercle bacilli become modified, either in cultural characters or in virulence, during long residence in the human skin. The total number of cases in which more than one examination was made was 32 . At the first investigation the results were as follows:

(a) 7 cases yielded fully virulent bovine strains.

(b) 17 cases yielded attenuated bovine strains.

(c) 1 case yielded a fully virulent human strain.

(d) 7 cases yielded attenuated human strains.

The results of reinvestigating the cases in each of these four groups are considered separately. 


\section{(a) Cases yielding fully virulent bovine strains on first examination}

The 7 cases in this group were reinvestigated 1-5 times. The intervals between the excision of material for examination in any given case ranged from $1 \frac{3}{4}$ to $7 \frac{1}{2}$ years, and the total number of strains investigated was 45. Most of the patients were receiving Finsen light treatment.

Culturally each of the 7 strains isolated initially was of the characteristic dysgonic type. The same held true of the strains isolated subsequently. No change in cultural behaviour was observed, even when changes in virulence were manifest.

The virulence of the different strains was tested on guinea-pigs, rabbits, calves, goats and sometimes monkeys. The number of animals injected was large, and in the investigation of the strains from one case alone over 100 animals were used. As it is manifestly impossible to describe in detail the lesions produced in each of these animals, I must be content with summarizing the general results.

In each case the strains of bovine bacilli became attenuated during the series of reinvestigations. In one case at the first investigation the bacilli were found to be attenuated at one site of the disease but not at another. In a second case the bacilli isolated 25 months after the initial investigation were fully virulent in some nodules and attenuated in others. In the remaining 5 cases attenuated bacilli were isolated at the second or later reinvestigation.

Three of the attenuated strains from 3 cases, one being a single colony strain, were restored to full virulence during their passage through the bodies of rabbits.

\section{(b) Cases yielding attenuated bovine strains on first examination}

The 17 cases in this group were reinvestigated at intervals ranging from 1 month to 15 years. Eleven of the cases were reinvestigated once, 4 twice, and 2 three times. Including the strains originally isolated, a total of 40 strains was studied. As in the preceding group, large numbers of animals were used for examining the virulence of the strains. The detailed findings in six of the cases were reported some years ago (Griffith, 1914b).

No evidence was obtained to show that attenuation was progressive. Four of the strains first isolated reacquired full virulence on passage through animals. When this happened, all strains subsequently isolated from the same case did so too. On the contrary, when the first strain could not be restored to full virulence, then neither could any of the later strains. In other words, once a strain had reached a certain degree of attenuation, it remained apparently fixed at this level of virulence.

No change occurred in the cultural characters of the bacilli.

\section{(c) Case yielding a fully virulent human strain on first examination}

There was only one case in this group. A fully virulent strain of human type was obtained from a lesion on the right elbow of a patient who had been suffering from lupus for 18 months. A year later a second strain was isolated from a lupus patch on the right knee; it appeared to be identical in both cultural characters and in virulence with the original strain. 


\section{(d) Cases yielding attenuated human strains on first examination}

Six of the 7 cases in this group were reinvestigated on one occasion only; from the remaining case material was re-examined on two occasions. The intervals between the first and last examinations ranged in these cases from 2 months to 8 years. The different strains from the same case were identical in cultural characters, and from 6 of the cases they appeared also to be identical in virulence. In the seventh case the second strain, isolated 10 months after the first, appeared to have undergone a slight further degree of attenuation.

The general conclusion to be drawn from these serial investigations of the same case is that tubercle bacilli do not continue to decline in virulence during the course of lupus; having reached a certain degree of attenuation, they remain stable for an indefinite period. In their cultural characters they seem to remain permanently stable; even in the two cases in which the disease had lasted for 30 and for 52 years, typical strains of bovine type were isolated from the tissues.

\section{Passage experiments}

Observations on the stability of virulence of modified bovine strains were numerous, because of the variety of available species of animals providing suitable environmental conditions for such a study. Passage experiments with human tubercle bacilli were less numerous, because the only readily available species of animal appropriate for the purpose was the guinea-pig.

\section{Bovine strains}

The general plan of the passage experiments was to inoculate an animal with a culture of an attenuated strain and, after a variable period of time ranging from about 6 weeks to 2 years, to recover the strain from a distal lesion. This strain was then used to inoculate another animal, or more usually a pair of animals, and again recovered and tested on two further animals. In this way many of the strains were passed through the bodies of a number of animals in series-up to 9 . The animals included guinea-pigs, rabbits, calves, goats and monkeys. The final passage culture was tested on two rabbits, and sometimes on other species as well. Forty strains were tested in all.

Analysis of the results showed that attenuated strains from 25 of the cases were not enhanced in virulence by passage. A feature of these experiments was the great variation in the extent of disease in different animals in any given series of passages: the lesions varied from slight tuberculosis to severe generalized tuberculosis. Repeatedly during the course of an experiment the result in a rabbit or a calf suggested that there had been an increase in virulence, but on further passage this was shown to be illusory, the final culture causing no more severe disease than had been produced by the original strain. The extensive lesions set up in some of the passage animals were presumably due to the specially high susceptibility of these animals rather than to an alteration in the intrinsic virulence of the strain. 
In 15 cases the bacilli, in their passage through the calf, the goat or the rabbit or through two or all three of these species, acquired the full virulence of the bovine type. Strains from 2 of the cases, which originally had produced chronic disease only in the susceptible guinea-pig and monkey, regained their full virulence in one passage and remained fully virulent through 4 or 5 successive passages. In the remaining 13 cases the increase in virulence did not always occur in the first passage, nor did it occur uniformly in all animals. Thus a strain would regain its full virulence in one species of animal, as judged by injection into rabbits and other animals when tested, but not in other species, the suggestion being that the conditions for recovery of virulence were more favourable in the tissues of some animals than of others. Contrary to expectation, one did not always find that strains recovered from severe and progressive lesions were fully virulent or that strains recovered from moderate and mainly retrogressive tuberculosis were attenuated.

The possibility that the apparent increase in virulence of a given strain was due to natural infection of an animal during the course of the experiment was continuously borne in mind. There was nothing, however, in the distribution of the lesions in the passage animals to suggest that this had occurred. Moreover, similar results were obtained with different strains from the same patient, isolated either at the same time or after a long interval of time.

\section{Human strains}

As already mentioned, experiments with attenuated human strains were less numerous than with attenuated bovine strains. Strains from 4 cases failed to regain their virulence by residence in the tissues of the guinea-pig for times ranging from 408 to 855 days. Likewise strains from a further 3 cases failed to regain their virulence by residence in the tissues of the monkey for times ranging from 100 to 312 days. From one monkey injected with an attenuated human strain a fully virulent human strain was isolated, but the anatomical distribution of the lesions plainly indicated a naturally acquired extraneous infection.

The conclusions to be drawn from these experiments are:

(1) Bovine tubercle bacilli that have become reduced in virulence through residence in human skin may or may not regain their virulence when subjected experimentally to prolonged residence in the animal body.

(2) The majority of attenuated bovine strains appear to have become fixed at a given level of virulence and cannot be changed by procedures of this nature.

(3) When attenuated bovine strains are altered by animal passage, they usually regain their full virulence.

(4) Human attenuated strains are apparently not restored to full virulence by prolonged sojourn in animal tissues, but their experimental investigation was carried out on a far smaller scale than that of the corresponding bovine strains. 
Comparison of strains from different lesions excised from the same patient on the same day

Material from 11 cases of generalized lupus was excised on the same day from different regions of the skin. Cultures were made from each specimen and compared for their growth characters and their virulence. The results are shown in Table 2.

Table 2. Comparison of strains from different lupus lesions of the same patient

\begin{tabular}{|c|c|c|c|}
\hline $\begin{array}{l}\text { Cultural } \\
\text { characters }\end{array}$ & $\begin{array}{c}\text { No. of } \\
\text { patients }\end{array}$ & $\begin{array}{l}\text { No. of } \\
\text { strains } \\
\text { studied }\end{array}$ & Virulence \\
\hline Dysgonic: & 6 & 12 & All attenuated bovine \\
\hline Dysgonic & 2 & 4 & $\begin{array}{l}\text { From each patient } 1 \text { strain fully } \\
\text { virulent bovine and } 1 \text { attenuated } \\
\text { bovine }\end{array}$ \\
\hline Dysgonic & 1 & 3 & $\begin{array}{l}2 \text { strains fully virulent bovine, } \\
\text { 1 slightly attenuated bovine }\end{array}$ \\
\hline Eugonic & 1 & 3 & All fully virulent human \\
\hline Eugonic & l & 3 & All strains attenuated human \\
\hline
\end{tabular}

The strains isolated from different regions of the skin of the same patient were identical in cultural characters. From the table it will be seen that, in 3 patients infected with the bovine type, strains differing in virulence were obtained from different sites.

In addition to these 11 cases, 5 cases were studied in which the patient was suffering from both lupus and scrofulodermia. Tubercle bacilli isolated from both lesions proved to be of the bovine type in 4 cases and of the human type in 1 case. The human strain and the bovine strains from two of the cases were attenuated. In the remaining 2 cases infected with the bovine type the strains from the lupus lesions were less virulent than those from the scrofulodermic abscesses, but they were isolated a year later.

\section{C'omparison of strains from lupus and from internal tuberculous lesions from the same patient}

Ten cases were studied in which the patient was suffering not only from lupus but from some internal lesion affecting the lymphatic nodes, peritoneum, lungs, bones and joints, or meninges. Cultures were made from these lesions and compared. The results are given in Table 3.

It will be seen that in 3 patients infected with the bovine type and in 2 patients infected with the human type the strains from the internal lesions were fully virulent whereas those from the lupus lesions were attenuated. In 1 of these patients the lupus strain was studied somewhat later than the strain from the sputum, but in 3 of the others the lupus strains were cultured before the material from the internal lesions. In these 5 cases the lupus had been present from $1 \frac{1}{2}$ to 17 years. It is probable that in some of them the internal lesions had been present. for an equal length of time, yet the bacilli in them had remained fully virulent. 
Table 3. Comparison of strains from lupus and from internal lesions of the same patient

\begin{tabular}{|c|c|c|c|}
\hline $\begin{array}{l}\text { Cultural } \\
\text { characters }\end{array}$ & $\begin{array}{l}\text { No. of } \\
\text { patients }\end{array}$ & $\begin{array}{l}\text { No. of } \\
\text { strains } \\
\text { studied }\end{array}$ & Virulence \\
\hline Dysgonic & 2 & 5 & All strains virulent bovine \\
\hline Dysgonic & I & 2 & Both strains attenuated bovine \\
\hline Dysgonic & 3 & 13 & $\begin{array}{l}\text { Internal strains virulent bovine, } \\
\text { lupus strains attenuated bovine }\end{array}$ \\
\hline Eugonic & 1 & 2 & Both strains virulent human \\
\hline Eugonic & 1 & 2 & Both strains attenuated human \\
\hline Eugonic & 2 & 5 & $\begin{array}{l}\text { Internal strains virulent human, } \\
\text { lupus strains attenuated human }\end{array}$ \\
\hline
\end{tabular}

\section{BACTERIOLOGICAL INVESTIGATION OF STRAINS FROM SCROFULODERMIA}

During the years 1917 and 1918 Dr H. A. Ellis of Middlesbrough sent me material from an unusual series of cases of cutaneous tuberculosis. Most of them were characterized by scrofulodermic abscesses in the skin and subcutaneous tissues, oceurring in different regions of the integument, sometimes single, more often multiple, and frequently accompanied by other forms of tuberculosis. In about half the cases the abscesses were situated in the neck and were associated with tuberculous cervical glands, though not stated to be directly connected with them. Tubercle bacilli were isolated from 52 of these cases and described in some detail in the Journal of Pathology and Bacteriology (Griffith, 1920). Later cultures were obtained from 8 further cases, making 60 in all, and these form the subject of the present brief report.

Table 4. Analysis of sixty cases of scrofulodermia

$\begin{array}{ccccc}\begin{array}{c}\text { Age period } \\ \text { in years }\end{array} & \begin{array}{c}\text { No. of } \\ \text { cases }\end{array} & \begin{array}{c}\text { Human } \\ \text { type }\end{array} & \begin{array}{c}\text { Bovine } \\ \text { type }\end{array} & \begin{array}{c}\text { Percentage } \\ \text { bovine } \\ \text { type }\end{array} \\ \text { Under } 5 & 15 & 7 & 8 & 53 \\ \overline{5}-15 & 30 & 17 & 13 & 43 \\ 16 \text { and over } & 15 & 14 & 1 & 7 \\ \text { Total } & 60 & 38 & 22 & 37\end{array}$

In Table 4 the cases are analysed to show the proportion of human to bovine infections at the different age periods in which the lesions are said to have appeared.

The numbers are small, but they show, as usual in this country, a higher proportion of bovine-type infections in the younger than in the older age groups.

Of the 38 culturally human strains, 30 were of full virulence, 1 appeared to be a little reduced in virulence, 4 were definitely attenuated, and 3 were not tested for virulence.

Of the 22 culturally bovine strains, 17 were fully virulent for rabbits and 5 were attenuated. 
Next to lupus, scrofulodermia stands highest among the different forms of human tuberculosis as a source of attenuated bacilli. The proportion, however, of attenuated to standard strains is very much lower in scrofulodermia than in lupus, being about one-sixth in the former and about two-thirds in the latter disease. In scrofulodermia the subcutaneous or the deeper layers of the skin are affected and the lesions are of relatively short duration, whereas in lupus the lesions are more superficial and of greater chronicity. These differences may perhaps explain why tubercle bacilli are more often attenuated in lupus than in scrofulodermia.

\section{v. DISCUSSION}

As shown in Table l (p. 15), 102 of the strains studied from cases of lupus were of the human and 102 of the bovine type. Of the human type approximately $60 \%$, and of the bovine type approximately $67 \%$, showed some degree of diminution in virulence. With one exception, that of a bovine strain which gave a moderately eugonic growth, all the strains could be clearly divided on cultural grounds into the human or the bovine type. In spite of their change in virulence, there was nothing to suggest that the attenuated strains were in process of transition from one type to the other; they represented rather members of the human or the bovine type that had undergone a modification in their pathogenic properties.

The attenuated bovine strains produced lesions in the differential group of animals-rabbit, calf and goat-that were more severe than those of the human type and characteristic of bacilli of the bovine type, only of greater chronicity. The strains formed a continuous series of varying grades of virulence merging at one end of the scale into the standard of the bovine type; and it was possible in some instances to raise their virulence to the standard level by passage through the bodies of animals. Similarly, the attenuated human strains clearly belonged to the human type, because their virulence for the rabbit, the calf and the goat was low, and because they gave rise to typical tuberculosis in guinea-pigs and monkeys. The effects produced in this latter group of animals differed from those following the inoculation of standard human strains only in their lesser severity. These strains likewise formed a continuous series of varying virulence merging at the upper end of the scale into the standard of the human type. In not a single instance, it may be emphasized, were culturally human and bovine types found together in the same patient.

The question may now be considered whether the modification in virulence of the bacilli was antecedent or subsequent to entry into the patient affected with lupus. All the evidence, in my view, favours the supposition that the modification took place in the skin during the course of the disease. The arguments may be put forward as follows:

(a) If the bacilli were already attenuated at the time of infection then it should be possible to find such attenuated forms among either human beings or animals responsible for conveying the disease.

Tubercle bacilli with reduced virulence, apart from lupus, are rare in tuberculosis of man. As will be seen from Table 5, 1 strain has been isolated from a 
cervical gland, 1 from an inter-muscular abscess, and 7 strains from bone and joint tuberculosis, but patients with these lesions are not infectious; and among the 2000 strains isolated from pulmonary tuberculosis, only 4 have been found of reduced virulence. Turning to animals, it may be said that organisms of this type are unknown in cattle or in their milk. In the horse, in which tuberculosis is an uncommon disease, about half the bovine-type strains are attenuated in virulence (Griffith, 1937), and in the pig attenuated strains have very occasionally been found; neither of these two animals, however, seems to be a likely source of infection for children. It may be added that lupus itself is not a source of infection, and that none of the cases referred to in this report was traceable to a previous case of lupus.

Table 5. Different kinds of aberrant strains found in human tuberculosis

$\begin{array}{lrrrrr} & \text { Yo. of } & \text { Attenuated Attenuated } & \text { Dysgonic } & \text { Eugonic } \\ \text { Form of tuberculosis } & \text { cases } & \text { human } & \text { bovine } & \text { human } & \text { bovine } \\ \text { Cervical gland } & 126 & 1 & - & 1 & 2 \\ \text { Inter-muscular abscess } & 2 & 1 & - & - & - \\ \text { Bone and joint } & 553 & - & 7 & 15 & - \\ \text { Meningeal } & 263 & - & - & 2 & - \\ \text { Genito-urinary } & 65 & - & - & 1 & - \\ \text { Autopsies } & 183 & - & - & 34 & - \\ \text { Pulmonary } & 2000 & - & 4 & \end{array}$

(b) Lupus is often secondary to cervical glandular tuberculosis and originates in the skin near a sinus or at the site of an operation for removal of the glands. In this series 24 patients gave a history of glandular tuberculosis before the lupus appeared: 16 of the 24 lupus strains proved to be attenuated but only 1 of the glandular strains.

(c) In several cases cultures were obtained from an internal tuberculous lesion as well as from the lupus lesion. In 5 of these the cultures from the deep-seated lesions were fully virulent, whereas those from the skin lesions were attenuated.

(d) In each of 11 cases two or more strains were obtained at the same time from lupus lesions on different parts of the body. In 3 of these the different strains, though identical in cultural characters, differed widely in virulence. These findings I interpret as evidence not of mixed infection with two races of bacilli differing in virulence, but as indicating that tubercle bacilli do not necessarily undergo modification of virulence simultaneously in different lesions.

(e) Though no close association could be demonstrated between the duration of the disease and the degree of attenuation of the bacilli, it is remarkable that most of the fully virulent strains came from young persons or from patients in whom the disease had lasted only a short time. Table 6 shows that the patients infected with strains of standard virulence, whether of the human or the bovine type, were on the average younger than those infected with attenuated strains, and that the average duration of the disease was shorter. This is particularly evident with the human strains ; in not a single patient who had suffered from the disease for 7 years or over was a fully virulent strain isolated. It is difficult to avoid the conclusion 
that modification in virulence is increasingly likely to occur with increasing duration of the disease.

$(f)$ This last conclusion is borne out by the results of certain serial investigations on the same cases that I was able to make. Seven cases which, when first examined bacteriologically, yielded fully virulent bovine strains were submitted $1 \frac{3}{4}-7 \frac{1}{2}$ years later to one or more further investigations. In each of these cases between the first and the last investigations the tubercle bacilli underwent a modification in virulence. As noted earlier in this paper, attenuation of a given strain does not appear to be progressive; the process seems to stop at a given level, which varies greatly from strain to strain.

Table 6. Relation of age and duration of disease to attenuation of human and bovine strains

\begin{tabular}{|c|c|c|c|c|c|c|}
\hline \multirow[b]{2}{*}{ Type of organism } & \multirow{2}{*}{$\begin{array}{l}\text { No. of } \\
\text { patients }\end{array}$} & \multicolumn{2}{|c|}{ Age in years } & \multirow{2}{*}{$\begin{array}{l}\text { No. of } \\
\text { patients }\end{array}$} & \multicolumn{2}{|c|}{$\begin{array}{c}\text { Duration in years, } \\
\text { when known }\end{array}$} \\
\hline & & $\overparen{\text { Range }}$ & $\overbrace{\text { A.M. }}$ & & Range & A.M. \\
\hline Human standard & 41 & $2-57$ & 12 & 27 & $0 \cdot 25-6$ & $2 \cdot 6$ \\
\hline Human attenuated & $60^{*}$ & $2-71$ & 21 & 52 & $0 \cdot 5-40$ & $12 \cdot 4$ \\
\hline Bovine standard & 34 & $3-39$ & 12 & 30 & $0 \cdot 5-33$ & $7 \cdot 1$ \\
\hline Bovine attenuated & 68 & $5-5 \overline{5}$ & 16 & 54 & $0 \cdot 25-52$ & $8 \cdot 8$ \\
\hline
\end{tabular}

\section{SUMMARY AND CONCLUSIONS}

1. Cultures of tubercle bacilli were obtained from 204 cases of lupus.

2. According to their cultural characters the strains could be divided into two main groups, one containing 102 strains with the characters of human tubercle bacilli and the other containing 102 strains with the characters of bovine tubercle bacilli.

3. Virulence tests were carried out on large number of animals, and in one instance over 100 animals were used for testing strains from a particular case.

4. Of the 102 culturally human strains 41 were highly virulent for the guineapig, and for the monkey when this was tested, producing progressive and rapidly fatal tuberculosis. The remaining 61 strains $(59.8 \%)$ showed varying degrees of attenuation.

5. Of the 102 culturally bovine strains 34 were highly virulent for all the animals tested, producing in them severe and rapidly fatal general tuberculosis identical with that set up by standard bovine bacilli. The remaining $68(66.7 \%)$ were of lower virulence for the rabbit, and for the calf and goat when these animals were tested, than standard bovine strains, but they all produced in one, two, or three of these species more extensive tuberculosis than human bacilli. For the guineapig, and for the monkey when tested, these strains fell below the virulence of standard bovine strains, except for a few whose virulence was only slightly lowered for the rabbit. 
6. A reinvestigation was made of 32 cases:

(a) In 7 cases originally yielding virulent bovine strains, later cultures obtained $1 \frac{3}{4}-7 \frac{1}{2}$ years after the first showed some degree of attenuation, though when multiple lesions were present this was manifest in only some of the strains.

(b) In 17 cases originally yielding attenuated bovine strains, reinvestigation on one, two, or three occasions over a period extending up to 15 years showed the presence of similarly attenuated bacilli; there was little or no evidence to suggest that attenuation had been progressive.

(c) One case infected originally with a virulent human strain yielded an identical strain a year later.

(d) In 7 cases originally yielding attenuated human strains, reinvestigation on one or two occasions up to 8 years later revealed the presence of similarly attenuated bacilli, except for one strain that appeared to be slightly less virulent than the original one.

From these findings it is concluded that attenuation is not continuously progressive but that any given strain, having reached a particular level of attenuation, tends to remain stable for an indefinite period.

7. With many of the attenuated bovine strains passage experiments were made in guinea-pigs, rabbits, calves, goats or monkeys to see if they could be restored to full virulence by contact with the internal tissues of a susceptible host. Of 40 attenuated bovine strains tested, 15 alone regained their full virulence. None of 7 attenuated human strains was restored to full virulence by passage through guinea-pigs or monkeys, even when residence in the tissues lasted for over 2 years. In the bovine series it was noted that when attenuated strains cultured from lupus patients in the early stage of the disease could be restored to full virulence by passage, then strains cultivated from the same patient months or years later reacted similarly.

These observations support the conclusion reached under $(6)$ that attenuation of a given strain does not progress beyond a certain point. When, on the other hand, the early strains remained attenuated on passage, the later strains did so too.

8. From 3 out of 9 patients suffering from multiple lupus lesions and infected with the bovine type, strains cultivated from different lesions on the same day differed in virulence, fully virulent and attenuated strains being found in separate lesions.

9. In 10 cases cultures were made simultaneously from lupus lesions and from internal tuberculous lesions from which the patients were also suffering. In 5 of these cases the lupus strains proved to be attenuated while the strains from the internal lesions were fully virulent.

10. In addition to the 204 cases of lupus, 60 cases of scrofulodermia were studied. From 38 patients bacilli of the human type and from 22 bacilli of the bovine type were cultivated. Of 57 strains tested in animals, $10(17.5 \%)$ were found to be attenuated in virulence.

11. Five cases were studied in which lupus and scrofulodermia co-existed. In 2 of these the scrofulodermia strains were fully virulent while the lupus strains were attenuated. 
12. The general conclusion is drawn that tuberculosis of the skin is often associated with the presence of human or bovine tubercle bacilli of attenuated virulence. In the present series $63.2 \%$ of the lupus and $17 \%$ of the scrofulodermia strains were attenuated. Such strains are rarely found in other forms of tuberculosis of man.

13. For reasons that are given under the discussion (pp. 22-24) it is believed that the attenuation of a high proportion of lupus strains is due to residence in the skin; no other tissue of the body seems to have this particular effect.

14. The observations recorded confirm the thesis that these atypical strains of tubercle bacilli are not separate types, but represent temporary or quasi-permanent alterations in virulence of strains of one or other of the two types infecting man.

15. It is clear that the cultural characters of tubercle bacilli are very much more stable than their virulence; in not a single case in which re-examination was made of the lupus lesion-often many years later--was a change in the cultural characters observed.

I am deeply indebted to the various medical men who have collaborated by sending material to me for examination. I am particularly grateful to the late Dr J. H. Sequeira and the late Sir Henry Gauvain who together supplied over half the cases of lupus, and to Dr H. A. Ellis who supplied nearly all the cases of scrofulodermia.

I should also like to thank the late Sir German Sims Woodhead who provided the necessary laboratory accommodation for me at Cambridge: the Grocers Company who awarded me a research scholarship for the first three years of my work there; and the Medical Research Committee and later the Medical Research Council who defrayed the expenses of the inquiry subsequently.

\section{REFERENCES}

(Griffith, A. S. (1914ı). Rep. L.G.B. publ. Hlth med. Subj., X.'. no. 88. p. 105.

('Riffith. A. S. (1914b). .J. Path. Baci. 18, Suppl. p. 591.

( ARIFFith, A. S. (1915). Lancet. 1, 1275.

(iniffith, A. s. (1920). J. Path. Bact. 23, 129.

('RIfFith, A. S. (1922). J.R. Inst. publ. Hlth, 30, 139.

Griffith, A. \$. (1924). Tubercle, Lond.. 5, 569.

(Griffith, A. S. (1932). Z. Tuberk. 64, 108.

('RIfFTth, A. S. (1937). J. comp. Path. 50, 159.

(iniffith, A. S. (1941a). Tubercle, Lond., 22, 33.

Giriffith, A. S. (1941b). J. Hyg.. C'amb.. 41, 272.

Report (1907). Royal Commission on Tuberculosis, 2nd Interim Report, Part II. Appendix. vols. 3 and 4 .

Report (1911). Ibid. Final Report. I'art II. Appendix. vols. 2 and 3.

(.MS. received for publication 12. III. 56) 